\title{
Loneliness \& Depression among Old Aged People
}

\author{
Dr. Farhat Jabin ${ }^{1 *}$
}

\section{ABSTRACT}

In the present research an attempt was made to study the relationship between loneliness and depression among old aged people. The study was carried on by formulating hypotheses that (a)There would be significant difference between loneliness and depression level of old aged people (b)-There would be positive correlation between loneliness and depression. For empirical verification of these two hypotheses a study was conducted on 60 old aged people of Patna and its neighboring places, by employing incidental-cum-purposive sampling technique. Their age ranged from 60 to 75 years. The UCLA Loneliness Scale was used for measuring loneliness. In order to check the level of depression in old aged people, Hindi adaptation of Beck's Depression Inventory was applied. For the analysis of the obtained scores t-ratio and co-efficient of correlation were computed. The result confirmed all the hypotheses. The findings on the whole indicated that there is significant difference between loneliness and depression level of old aged people. Positive correlation between loneliness and depression level was also observed by the researcher. The findings of the present research reveal that loneliness plays an important role in determining depression among old aged people. Depression level can be reduced by reducing loneliness among old aged people.

Keywords: Loneliness, Depression, Old-Age

Seasons of life are the offerings of the dynamic creation of God. Each developmental phase has its unique characteristic features, developmental tasks, virtues and realization points in the platform of positive psychology. Old age is the final developmental phase which is adorned by folds of wisdom, generatively and ripened meaning of life for successful aged. Since the dawn of civilization, human beings have recognized a progression through the life course, from infancy through old age. One cannot fully understand what old age means unless one understands it as part of the entire course of human life, and this approach is called the life course or life-span perspective (Settersten, 2003).

\footnotetext{
${ }^{1}$ Dept. Of Psychology, Magadh Mahila College, Patna University, Patna, India *Responding Author

(C) 2016 I F Jabin; licensee IJIP. This is an Open Access Research distributed under the terms of the Creative Commons Attribution License (http://creativecommons.org/licenses/by/2.0), which permits unrestricted use, distribution, and reproduction in any Medium, provided the original work is properly cited.
} 


\section{Loneliness \& Depression among Old Aged People}

Old age is the closing period in the life span. It is a period when people "move away" from previous, more desirable period or time of "usefulness". Adjusting to the changes that accompany old age requires that an individual is flexible and develops new coping skills to adapt to the changes that are common to this time in their lives (Warnick, 1995).

The increased span of life in India, a person over 65 years of age may be considered as aged. India is the second largest population of elderly (60+) in the world. The population of seniors in India has grown from 3.3 percent in 1950 to 7.6 percent in 2000. It is predicted that by 2050 a whopping 15.1 percent of Indian population would consist of individuals 65 years and older (United Nations, 1998; United Nations Population Division, 1999). The increase in the number of elderly individuals has led to discussions about health care facilities and social support system in India. Aging is usually associated with physical decline and frailty as well as psychological modifications. A number of research studies focus on structural and functional decrements (Margrain and Boulton, 2005; Solomon, Shock and Aughenbaugh, 1970; Singh, 2004; Ramamurti, 2002). Powerlessness or perception of others in control can be an emotional risk factor in old age too (Kunzmann, Little and Smith, 2002).

There is a growing body of evidence that suggests that psychological and sociological factors have a significant influence on how well individuals age. Aging research has demonstrated a positive correlation of someone's religious beliefs, social relationships, perceived health, selfefficacy, socioeconomic status and coping skills, among others, with their ability to age more successfully.

The question comes to mind as to what successful aging is and how it can be achieved in the face of such decline and changes. According to Wong (1989; 2000), prolonging life without providing any meaning for existence is not the best answer to the challenge of aging. He defines successful aging as a relatively high level of physical health, psychological well-being and competence in adaptation (Wong, 1989). However, Wong (1989; 2000) notes that the latter two aspects are more important than physical health when determining successful aging. Competence in adaptation emphasizes personal responsibility, implying that an individual has a choice of how she will cope with the challenges and changes that occur with growing older such as retirement, physical decline, personal losses, suffering and impending death. This view is supported by numerous other studies too (Rowe \& Khan, 1998; Fisher and Specht, 1999).

Depression or the occurrence of depressive symptomatology is a prominent condition amongst older people, with a significant impact on the well-being and quality of life. Many studies have demonstrated that the prevalence of depressive symptoms increases with age (Kennedy, 1996). Depressive symptoms not only have an important place as indicators of psychological well-being but are also recognized as significant predictors of functional health and longevity. It interferes with an individual's ability to function and causes great distress to him/her (Geist and Jefferson, 


\section{Loneliness \& Depression among Old Aged People}

1997). Late-life depression has been shown to be influenced by genetic, situational, biological and psychosocial factors. Longitudinal studies demonstrate that increased depressive symptoms are significantly associated with increased difficulties with activities of daily living (Penninx et al., 1998). Community-based data indicate that older persons with major depressive disorders are at increased risk of mortality (Bruce, 1994). There are also studies that suggest that depressive disorders may be associated with a reduction in cognitive functions (Speck et al., 1995).

Depression has a causal link to numerous social, physical and psychological problems. These difficulties often emerge in older adulthood, increasing the likelihood of depression; yet depression is not a normal consequence of these problems. When the onset of depression first occurs in earlier life, it is more likely that there are genetic, personality and life experience factors that have contributed to the depression. Depression that first develops in later life is more likely to bear some relationship to physical health problems. An older person in good physical health has a relatively low risk of depression. Physical health is indeed the major cause of depression in late life. There are many reasons for this, which include the psychological effects of living with an illness and disability, the effects of chronic pain; the biological effects of some conditions and medications that can cause depression through direct effects on the brain; and the social restrictions that some illnesses place upon older people's life style resulting in isolation and loneliness.

There are strong indications that depression substantially increases the risk of death in adults, mostly by unnatural causes and cardiovascular disease (Wulsinet al., 1999). Some populationbased studies did find that this independent relationship does exist in later life, while others did not.

Loneliness is a subjective, negative feeling related to the person's own experience of deficient social relations. Loneliness has been defined in the social psychological literature as consisting of "emotional isolation, which results from the loss or lack of a truly intimate tie ..and social isolation, the consequence of lacking a network of involvements with peers of some sort." (Weiss, 1973: p. iv) Thus, the experience of loneliness includes both the emotional pain of losing a loved one as well as a sense of exclusion or marginality from social ties. High levels of loneliness have been linked to higher risks of mental illness and suicide (Peplau and Perlman, 1982; Weiss, 1973).The determinants of loneliness are most often defined on the basis of 2 causal models. The first model examines the external factors, which are absent in the social network, as the root of the loneliness; while the second explanatory model refers to the internal factors, such as personality and psychological factors.

Loneliness may lead to serious health-related consequences. It is one of the 3 main factors leading to depression (Green et al., 1992), and an important cause of suicide and suicide 


\section{Loneliness \& Depression among Old Aged People}

attempts. A study carried out by Hansson et al. (1987) revealed that loneliness was related to poor psychological adjustment, dissatisfaction with family and social relationships.

As people grow old, the likelihood of experiencing age-related losses increases. Such losses may impede the maintenance or acquisition of desired relationships, resulting in a higher incidence of loneliness. Many people experience loneliness either as a result of living alone, a lack of close family ties, reduced connections with their culture of origin or an inability to actively participate in the local community activities. When this occurs in combination with physical disablement, demoralization and depression are common accompaniments. The negative effect of loneliness on health in old age has been reported by researchers (Heikkinen et al., 1995). The death of spouse and friends and social disengagement after leaving work or a familiar neighbourhood are some of the ubiquitous life-changing events contributing to loneliness in older people. Those in the oldest age cohort are most likely to report the highest rates of loneliness, reflecting their increased probability of such losses.

A study by Max et al. (2005) revealed that the presence of perceived loneliness contributed strongly to the effect of depression on mortality. Thus, in the oldest old, depression is associated with mortality only when feelings of loneliness are present. Depression is a problem that often accompanies loneliness. In many cases, depressive symptoms such as withdrawal, anxiety, lack of motivation and sadness mimic and mask the symptoms of loneliness.

Many people experience loneliness and depression in old age, either as a result of living alone or due to lack of close family ties and reduced connections with their culture of origin, which results in an inability to actively participate in the community activities. With advancing age, it is inevitable that people lose connection with their friendship networks and that they find it more difficult to initiate new friendships and to belong to new networks. . Depression and loneliness are considered to be the major problems leading to impaired quality of life among elderly persons. The present study was conducted to investigate the relationships among depression and loneliness in elderly people.

\section{The main objectives of the present study are:}

1. To assess the level of depression among elders.

2. To examine the degree of loneliness among old aged people.

3. To identify the relationship between depression and loneliness in elders.

\section{Hypotheses}

Keeping the objective in view following hypotheses were formulated for verification:

1. It was hypothesized that there would be significant difference between loneliness and depression level of old aged people. The formulation of this hypothesis was framed keeping in mind the assumptions on loneliness and depression. Through the literature 


\section{Loneliness \& Depression among Old Aged People}

review it was found that depression was related to the loneliness among old aged people. Hence, the focus of this study is to examine the difference between loneliness and depression among elders.

2. It was hypothesized that there would be positive correlation between loneliness and depression. This hypothesis was framed through literature review. Several studies attribute a positive relationship between loneliness and depression (Adams et al. 2004; Pressman et al. 2005; Cacioppo, Hughes, Waite, Hawkley \& Thisted, 2006; Blazer, D.G., 2002; Hawkley, Burleson, Berntson \& Cacioppo, 2003). Through the literature review it was found that higher level of loneliness were associated with more depressive symptoms in older adults. The inclusion of this hypothesis in the present research was to see that whether level of depression among older people is influenced by loneliness or not.

\section{METHOD}

\section{Sample}

The present investigation was carried on the 60 old people (Male \& Female both) of Patna and its neighbouring places by employing incidental-cum-purposive sampling technique. Their age ranged from 60 to 75 years. They were matched as far as possible in other respects.

\section{Tools}

$>$ A Personal Data Sheet prepared by the researcher was used for collecting necessary personal information about the respondents.

$>$ Beck Depression Inventory (Hindi adaptation by Sinha and Kapoor) was employed for measuring depression of old aged people.

$>$ The UCLA Loneliness Scale, (University of California, Los Angles) was used for measuring loneliness among elders.

\section{Procedure}

The test was administered in two different sessions. First of all personal data sheet was administered to the respondents and relevant information's were gathered. Thereafter, Beck Depression inventory and UCLA Loneliness Scale were administered to the respondents one by one with a short interval and the responses made by the subjects were scored, tabulated and analyzed by applying t-test and correlation-coefficient test. The findings based on these statistical techniques have been shown in table 1-3.

\section{RESULTS AND DISCUSSION}

For deriving useful conclusion, the data is organized in a systematic way. The obtained result is explained on the basis of statistical analysis. 
Loneliness \& Depression among Old Aged People

Table - 1, Mean value and SD of Loneliness \& Depression among Old Aged People

\begin{tabular}{|l|l|l|}
\hline $\mathbf{N}=\mathbf{6 0}$ & Mean & SD \\
\hline Loneliness & 31.28 & 12.35 \\
\hline Depression & 26.85 & 8.42 \\
\hline
\end{tabular}

The inspection of table -1 shows that the obtained mean value on loneliness and depression is 31.28 and 26.85 respectively. The obtained value of SD of loneliness and depression is 12.35 \& 8.42 respectively which clearly shows that in case of both loneliness and depression there is less internal variation because the obtained value of SD ( 12.35 ) and ( 8.42 ) is quite less to its obtained mean value ( 31.28 ) and ( 26.85 ) respectively. So, it can be said that as the degree of loneliness gets higher the level of depression will also be high which supports the hypothesis formulated by the researcher that " There would be significant difference between loneliness and depression level of old aged people ". Hence, the result of this study are consisted with that of Max et al. (2005) who revealed that the presence of perceived loneliness contributed strongly to the effect of depression on mortality. Thus, in the oldest old, depression is associated with mortality only when feelings of loneliness are present. Depression is a problem that often accompanies loneliness.

In order to verify the above hypothesis statistically and to further elaborate the findings t-ratio was applied by the researcher and thus the significant difference between two means was examined. The obtained results were recorded in the table-2.

Table - 2, Differences Between The Mean Scores Of Depression And Loneliness Among Old Aged People $(\mathrm{N}=60)$

\begin{tabular}{|l|l|l|l|l|l|l|l|}
\hline & N & Mean & SD & SE & t-ratio & df & p-value \\
\hline Loneliness & \multirow{2}{*}{6} & 31.28 & 12.35 & 1.59 & \multirow{2}{*}{2.04} & \multirow{2}{*}{58} & $\mathrm{P}<.05$ \\
\cline { 1 - 6 } & & 26.85 & 8.42 & 1.09 & & & \\
\hline
\end{tabular}

It is evident from table-2 that the difference between the mean scores of loneliness ( 31.28 ) and depression ( 26.85 ) is significant at .05 level of confidence. Thus, it can be concluded that there is significant difference between loneliness and depression level of older people. The obtained mean value of loneliness of the respondents is 31.28 which is higher than the obtained mean value 26.85 on depression. Hence, it is clear that the degree of loneliness is higher in comparison to depression level. This finding supports the hypothesis framed by the researcher. Though the obtained t-value $=2.04$ is significant but it is marginally significant, therefore, it can be concluded that the hypothesis framed by the researcher is partially confirmed. 


\section{Loneliness \& Depression among Old Aged People}

In order to test and verify the second hypothesis framed by the researcher that there would be positive correlation between loneliness and depression, coefficient of correlation was applied. Table -3 presents the summary of the coefficient of correlation.

Table - 3, Table Showing Coefficient Of Correlation Between Loneliness and Depression Among Old Aged People

\begin{tabular}{|l|l|l|l|l|}
\hline & N & r & df & p-value \\
\hline Loneliness & 60 & 0.95 & 58 & $\mathrm{P}<.01$ \\
\hline Depression & & & & \\
\hline
\end{tabular}

Table-3 reveals that the obtained value of coefficient of correlation (r) $=0.95$ is positive and highly significant at 0.01 level of confidence on df-58. This supports the hypothesis which suggests that there is a positive relation between loneliness and depression level of old aged people.

A study of all the above tables presents an extensive picture of the relationship between loneliness and depression level among elder people. All the hypotheses framed by the researcher are found statistically significant at .05 and .01 level of confidence respectively. The findings on the whole indicated that there is significant difference between loneliness and depression level of old aged people. Positive correlation between loneliness and depression level was also observed by the researcher. The findings of the present research reveal that loneliness plays an important role in determining depression among old aged people. Loneliness, coupled with other physical and mental problems, gives rise to feelings of depression in the elderly persons. A positive correlation between loneliness and depression is in accordance with the results obtained in literature (Green et al., 1992).

\section{RECOMMENDATION}

The findings of the present research thus have brought many new facts in respect to the relation between loneliness and depression level among elders. The findings of the present researcher have indicated that depression level of older people is very much affected by the presence of loneliness. Depression level can be reduced by reducing loneliness among old aged people.

Hence, to draw the curtain close, it may be stated that everything in this dynamic world has some advantages and limitations. Old age is no exception. There can be multiple reasons to feel disheartened like retirement, loss of loved ones, physical decline, loss of power and autonomy, and interpersonal conflicts. Successful aging can never be based on the denial of real losses as regards the quality of functioning in the last stage of life. Rather, the idea of successful aging involves graceful acceptance of reality as it is, adopting a generativity-glimmered orientation

(C) The International Journal of Indian Psychology, ISSN 2348-5396 (e)| ISSN: 2349-3429 (p) | 143 


\section{Loneliness \& Depression among Old Aged People}

towards life in general. Successful aging means growing old with good health and functional capacity, self-acceptance, autonomy, and forgiveness, engaging in learning and activities promoting personal growth, prosocial behaviour, healthy interactions with the family, deriving a sense of purpose or meaning in life and being satisfied with life. As such, the discourse may end with the notion that elderly individuals need to be accepted, respected, and provided with their requisite self-respect. This is likely to facilitate the elderly individuals to glance at life through the lenses of "experientially-rimmed wisdom" and accept factual realities gracefully, thereby helping them to bask in the "rays" of successful aging.

There are certain limitations in the study:

1. The sample size is restricted to few elderly persons. Hence in future, a similar study needs to be conducted on a larger section of the elderly population.

2. For determining gender differences, both male and female constituents of the sample should be equivalent in all respects.

3. Moreover, no formal diagnosis of depression was made in the sample used in the study. Self-report inventory was used for determining the level of depressive symptoms in the elderly persons.

Keeping in view the above limitations, longitudinal studies on a larger group of elderly men and women are needed in future. Thus the findings would stimulate other researchers also to take up such problems for further investigation.

For the purpose of general screening of the depression level among old aged people, the findings can be of much helpful to the counsellors, clinicians and psychologists. The findings would also help the researchers and youngsters.

\section{Acknowledgments}

The author appreciates all those who participated in the study and helped to facilitate the research process.

\section{Conflict of Interests}

The author declared no conflict of interests.

\section{REFERENCES}

Adam, K. B., Sanders, S., \& Auth, E. A. (2004). Loneliness and depression in independent living retirement communities : Risk and resilience factors [ Electronic version ]. Aging \& Mental Health, 8, 475-478.

Blazer, D. G. (2002). Self -efficacy and depression in late life : A primary prevention proposal [Electronic version]. Aging \& Mental Health, 6, 315-324. 


\section{Loneliness \& Depression among Old Aged People}

Bruce M. L, Seeman T, Merrill S. S, Blazer D. G. The impact of depressive symptomatology on physical disability: MacArthur Studies of successful aging. American Journal of Public Health. 1994;84:1796-1799. [PMC free article] [PubMed]

Cacioppo, J. T., Hughes, M. E., Waite, L. J., Hawkley, L. C., \& Thisted, R. A. (2006). Loneliness as a specific risk factor for depressive symptoms : Cross-sectional and longitudinal analyses, Psychology \& Aging, 21, 140-151.

Fisher, B.J., and Specht, D. (1999): Successful aging and creativity in later life, Journal of Aging Studies, 13(4): 457-472.

Geist, J.H. and Jefferson, J.W. (1992): Depression and its Treatment, Warner Communications Co. USA: New York.

Green B. H, Copeland J. R, Dewey M. E, Shamra V, Saunders P. A, Davidson I. A, Sullivan C, McWilliam C. Risk factors for depression in elderly people: A prospective study. ActaPsychiatr Scand. 1992; 86(3):213-7. [PubMed]

Hansson R. O, Carpenter B. N. Relationships in Old Age: Coping with the challenge of transition. New York, NY: Guilford Press; 1994.

Hansson R. O, Jones W. H, Carpenter B. N, Remondet J. H. International Journal of Human Development. 1986-1987; 27(1):41-53.

Hawkley, L. C., Burleson, M. H., Berntson, G. G., \& Cacioppo, J. T. (2003). Loneliness in everyday life : Cardiovascular activity, psychosocial context and health behaviours [Electronic version]. Journal of Personality and Social Psychology, 85, 105-120.

Heikkinen R, Berg S, Avland K. Depressive symptoms in late life. Journal of Cross Cultural Gerontology. 1995;10:315-330. [PubMed]

Kennedy G.J. The epidemiology of late-life depression. In: Kennedy G. J, editor. Suicide and depression in late life: Critical issues in treatment, research and public policy. New York: John Wiley and Sons; 1996. pp. 23-37.

Kunzmann, U., Little, T., Smith, J. (2002): Perceiving Control: A Double-Edged Sword in Old Age, The Journals of Gerontology Series B: Psychological Sciences and Social Sciences, 57: 484-491.

Margrain, T.H. and Boulton, M. (2005): Sensory Imapairment, in M.L. Johnson, V.L. Benston, P.G. Coleman, T.B.L. Kirkwood (Eds.), The Cambridge Handbook of Age and Ageing, Cambridge University Press.

Max L. S, David J. V, Jacobijn G, Aartjan T. F, Ross van der Mast \& Rudi G. J. Is depression in old age fatal only when people feel lonely? American Journal Psychiatry. 2005;162:178180. [PubMed]

Penninx B. W, Guralnik J. M, Ferrucci L, Simonsick E. M, Deeg D. J, Wallace R. B. Depressive symptoms and physical decline in community-dwelling older persons. Journal of the American Medical Association. 1998;127:1720-1726. [PubMed]

Peplau, L.A. and D. Perlman (Eds.). 1982. Loneliness: A Sourcebook of Current Theory, Research and Therapy. New York: John Wiley and Sons. 


\section{Loneliness \& Depression among Old Aged People}

Pressman, S. D., Cohen, S., Miller, G., Barkin, A., Rabin, B. S., \& Treanor, J.J. (2005). Loneliness, social network size and immune response to influenza vaccination in college freshmen [Electronic version]. Health Psychology, 24, 297-306.

Ramamurti, P.V. (2002): Intergenerational Relations, in K.R. Gangadharan (Ed.), Ageing in India: Emerging Trends and Perspectives (pp. 24-26), Heritage Hospital, Hyderabad.

Rowe, J.W. and Kahn, R.L. (1998): Successful Aging, New York: Pantheon Books.

Settersten, R.A., Jr. (Ed.) (2003): Invitation to the life course: 'Toward new understandings of later life, Amityville, NY:

Singh, S. (2004): Intergenerational Relationship: Study of Life Satisfaction, Attitude, Role and Expectation, M.A. Project Report, Department of Psychology, University of Delhi.

Solomon, N., Shock, N.W. and Aughen Baugh, P.S. (1970): The Biology of Aging, in A.M. Hoffman (Ed.), The Daily Needs and Interests of Older People (pp. 195-208), Springfield: Charles C. Thomas.

Speck C. E, Kukull W. A, Brenner D. E, Bowen J. D, McCormick W. C, Teri W. C, Pfanschmidt M. L, Thompson J. D, Larson E. B. History of depression as a risk factor for Alzheimer's disease. Epidemiology. 1995;6:366-369. [PubMed]

United Nations, World Population Prospects: The 1998 Revision, Vol 1: Comprehensive Tables, United Nations Population Division, Department of Economic and Social Affairs, NewYork, 1999.

Warnick J. Listening with different ears: Counseling people over sixty. Ft. Bragg CA: QED Press; 1995.

Weiss, R.S. 1973. Loneliness: The Experience of Emotional and Social Isolation. Cambridge: MIT Press.

Wong, P.T.P. (1989): Successful aging and personal meaning, Canadian Psychological, 30: 516525.

Wong, P.T.P. (2000): Meaning of life and meaning of death in successful aging, in A. Tomer (Ed.), Death attitudes and the older adult: Theories, concepts and applications (pp. 2336), Washington, DC: Taylor-Francis.

Wulsin L. R, Vaillant G. E, Wells V. E. A systematic review of the mortality of depression. Psychosomatic Medicine. 1999;161:6-17. [PubMed]

How to cite this article: F Jabin (2016), Loneliness \& Depression among Old Aged People, International Journal of Indian Psychology, Volume 3, Issue 4, No. 59, ISSN 2348-5396 (e), ISSN: 2349-3429 (p), DIP: 18.01.071/20160304, ISBN: 978-1-365-26307-1 